$16^{\text {th }}$ International Congress of Metrology, 11009 (2013)

DOI: $10.1051 /$ metrology/201311009

(C) Owned by the authors, published by EDP Sciences, 2013

\title{
MISE AU POINT D’UNE PLATEFORME D’ETALONNAGE DES COMPAS MAGNETIQUES
}

\author{
M. Le Menn, A. Lusven, E. Bongiovanni, P. Le Dû, D. Rouxel, S. Lucas, L. Pacaud
}

SHOM, 13 rue du Chatellier, CS 92803, 29228 Brest Cedex

\begin{abstract}
Instruments used to measure speed and direction of sea currents are equipped with magnetic compass and tilt sensors, in order to be able to reference their data in a terrestrial referential. Compass being sensible to their magnetic environment, it is necessary to calibrate currentmeters in their using configuration. In this order, a non-magnetic platform has been built. Reference directions and tilt expended uncertainties reached with this equipment are respectively $1^{\circ}$ and $0.32^{\circ}$. Results obtained during different trials of current profilers are exposed.
\end{abstract}

\section{Introduction}

La vitesse et la direction des courants marins étaient obtenues initialement avec des courantomètres à rotor. Cette technologie a été remplacée progressivement par des technologies acoustiques. On distingue :

- les courantomètres acoustiques qui mesurent l'effet Doppler dans une tranche d'eau par l'envoi d'impulsions et l'écoute des échos,

- des profileurs Doppler (ADP ou ADCP pour Acoustic Doppler Current Profiler) qui mesurent des vitesses dans des tranches d'eau réparties sur quelques mètres à plusieurs centaines de mètres.

$$
\left[\begin{array}{l}
V_{x} \\
V_{y} \\
V_{z}
\end{array}\right]=\left[\begin{array}{ccc}
\frac{2}{3 \sin (\beta)} & \frac{-1}{3 \sin (\beta)} & \frac{-1}{3 \sin (\beta)} \\
0 & \frac{-1}{\sqrt{2} \sin (\beta)} & \frac{1}{\sqrt{2} \sin (\beta)} \\
\frac{1}{3 \cos (\beta)} & \frac{1}{3 \cos (\beta)} & \frac{1}{3 \cos (\beta)}
\end{array}\right]\left[\begin{array}{l}
V_{1} \\
V_{2} \\
V_{3}
\end{array}\right]
$$

Courantomètres et profileurs mesurent des vitesses ( $V_{l}$, $V_{2}, V_{3}$ ) dans l'axe de leurs faisceaux (1). Leurs émetteurs sont inclinés à $20^{\circ}, 25^{\circ}$ ou $30^{\circ}$ (angle $\beta$ ). Connaissant la valeur de $\beta$, on peut calculer des vitesses $\left(V_{x}, V_{y}, V_{z}\right)$ dans leur repère propre.

Ils sont équipés en plus de compas du type 'flux-gate' pour retrouver la valeur des courants $(U, V, W)$ par rapport au Nord magnétique (angle $\Omega$ ), puis, connaissant la déclinaison magnétique, par rapport au Nord géographique (2). Ils sont, de plus, corrigés en inclinaison à l'aide de capteurs de 'tilt' qui mesurent les angles $\Psi$ et $\theta$ de roulis et tangage (dans l'équation (2), $C=\cos$ et $S=$ $\sin )$ :

\footnotetext{
$\bar{a}$ Corresponding author: marc.lemenn@SHOM.fr
}

$$
\left[\begin{array}{c}
U \\
V \\
W
\end{array}\right]=\left[\begin{array}{ccc}
C_{\psi} C_{\Omega} & \left(-S_{\psi} S_{\theta} C_{\Omega}+C_{\theta} S_{\Omega}\right) & \left(S_{\psi} C_{\theta} C_{\Omega}+S_{\theta} S_{\Omega}\right) \\
-C_{\psi} C_{\Omega} & \left(S_{\psi} S_{\theta} S_{\Omega}+C_{\theta} S_{\Omega}\right) & \left(-S_{\psi} C_{\theta} S_{\Omega}+S_{\theta} C_{\Omega}\right) \\
-S_{\psi} & -C_{\psi} S_{\theta} & C_{\psi} C_{\theta}
\end{array}\right]\left[\begin{array}{c}
V_{x} \\
V_{y} \\
V_{z}
\end{array}\right]
$$

L'exactitude de ces instruments, affichée par les constructeurs, est de 1 à $2 \%$ de la valeur mesurée en vitesse, $2^{\circ}$ en direction et $0,2^{\circ}$ en inclinaison. Quant à la répétabilité elle est de 0,5 à $1 \mathrm{~cm} / \mathrm{s}, 0,5^{\circ}$ en direction et $0,1^{\circ}$ en inclinaison.

Les compas sont composés de 3 magnétomètres montés orthogonalement qui mesurent des inductions $M_{x}, M_{y}$ et $M_{z}$. L'angle de cap est déterminé en établissant le rapport de leurs projections $M_{x h}$ et $M_{y h}$ dans le plan horizontal [1], à l'aide des angles mesurés par le capteur de tilt :

$$
\left[\begin{array}{l}
M_{x H} \\
M_{y H}
\end{array}\right]=\left[\begin{array}{ccc}
\cos \theta & \sin \theta \cdot \sin \psi & -\cos \psi \cdot \sin \theta \\
0 & \cos \psi & \sin \psi
\end{array}\right] \cdot\left[\begin{array}{l}
M_{x} \\
M_{y} \\
M_{z}
\end{array}\right]
$$

Les compas sont sensibles aux perturbations du champ magnétique engendrées par les éléments métalliques disposés à proximité. On distingue, les matériaux magnétiques 'durs' qui génèrent un champ permanent (aimants, électro-aimants...), des matériaux magnétiques 'doux' qui génèrent un champ sous l'action d'un autre champ.

Pour pallier ces défauts, les constructeurs mettent à disposition des procédures dites 'd'autocalibration', où l'utilisateur fait tourner l'instrument sur lui-même et le logiciel de l'instrument calcule, à l'aide le plus souvent d'algorithmes de calcul de moindres carrés itératifs, des coefficients de correction du compas. Problèmes, aucune 
référence ne permet de juger de la qualité de cet autoétalonnage, sur laquelle les constructeurs sont assez silencieux, rien n'est proposé dans les logiciels pour corriger les capteurs d'inclinaison, et, il n'existe aucune installation en France pour réaliser ce genre de mesure.

\section{Conception de la plateforme d'étalonnage}

Les courantomètres étant le plus souvent montés dans des cages de mouillage avec divers autres instruments, il est nécessaire d'étalonner leurs compas dans l'environnement d'utilisation. En conséquence, la plateforme doit pouvoir supporter des charges de l'ordre de $800 \mathrm{~kg}$.

Son lieu d'implantation a été déterminé après réalisation d'une cartographie magnétique pour délimiter une zone où les gradients magnétiques sont $<$ à $5 \mathrm{nT} / \mathrm{m}$. Sa conception fait suite à des expériences de faisabilité réalisées en 2007 [2].

Dans la zone présentant les gradients les plus faibles, une dalle en béton d'une bonne planéité a été fabriquée : elle possède une inclinaison maximale évaluée à $3 \mathrm{~mm} / \mathrm{m}$ soit $0,18^{\circ}$ et une rugosité moyenne équivalant à $0,15^{\circ}$ d'incertitude. Puis, un plateau tournant et inclinable a été conçu (Fig. 1). L'inclinaison du plateau est mesurée à l'aide d'un rapporteur numérique étalonnable dans un laboratoire de référence avec une incertitude type de $0,12^{\circ}$. Ces sources d'incertitude étant indépendantes, leur somme quadratique donne une incertitude élargie sur les inclinaisons de référence de $0,32^{\circ}$.

Après 1'installation de cette plateforme, une nouvelle cartographie magnétique a été réalisée pour s'assurer de l'absence d'anomalies.

Figure 1 : Plateforme d'étalonnage avec son plateau tournant, équipé d'une cage du type 'tripode' et d'un ADP. Station Sentinel au premier plan.

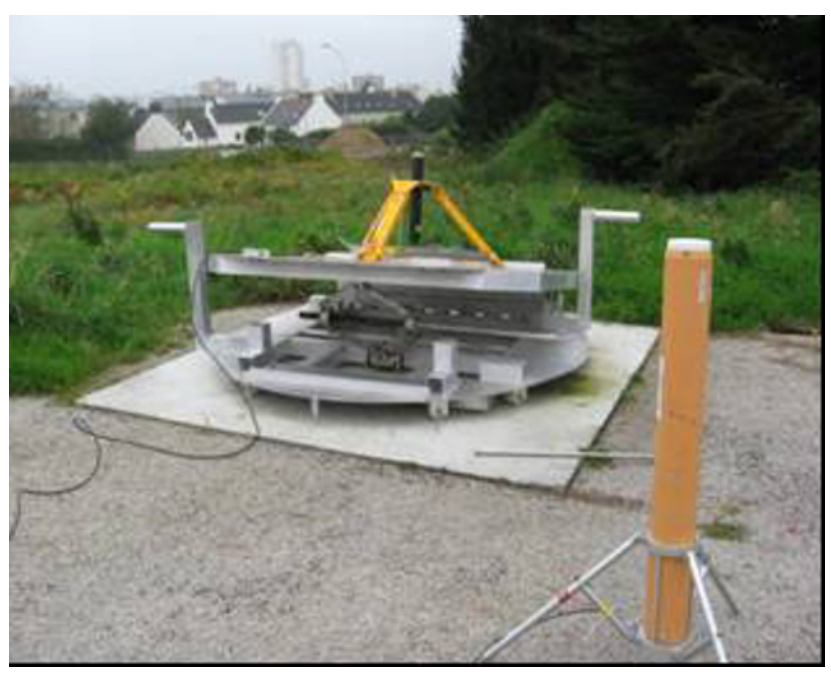

Les anomalies magnétiques sont calculées après correction des variations séculaires du champ (retrait du modèle global IGRF ou International Geomagnetic Reference Field). Sur ce site, elles présentent une pente croissante de $3,8 \mathrm{nT} / \mathrm{m}$ dans la direction Nord-Ouest Sud-Ouest, et une pente croissante de $3 \mathrm{nT} / \mathrm{m}$ dans la direction Nord - Sud, ce qui est conforme à notre tolérance. Ramené en déviation angulaire, cela correspond à une incertitude de 0,09 ․ L'effet des variations temporelles du champ magnétique terrestre a également été mesuré, sur 5 jours, à l'aide d'une station de référence Sentinel. Il correspond à une incertitude de $0,30^{\circ}$. Enfin, les variations de déclinaison magnétique ont été évaluées à $0,14^{\circ}$ Est/an, à l'aide du modèle WMM2010. Ces sources d'incertitude étant indépendantes, l'incertitude élargie liée à l'environnement magnétique peut être établie par une simple somme quadratique qui donne $0,69^{\circ}$.

Connaissant l'environnement magnétique, il faut ensuite étalonner en direction. Pour cela, la dalle béton a été repérée à l'aide d'un récepteur GPS Leica utilisé en mode RTK (Real Time Kinematic) (mesure différentielle à partir d'une station de référence). Les coordonnées du centre de la plateforme et d'un pilier de référence ont été repérées (mesures dans le système RGF-93 et passage dans le système altimétrique NGF-IGN69, coordonnées planes). Le gisement de cet axe de référence a été calculé et, connaissant la convergence locale du méridien, la direction du Nord géographique $\left(N_{v}\right)$, ou azimut, en a été déduite (Fig. 2).

Figure 2 : principe de l'étalonnage en direction.

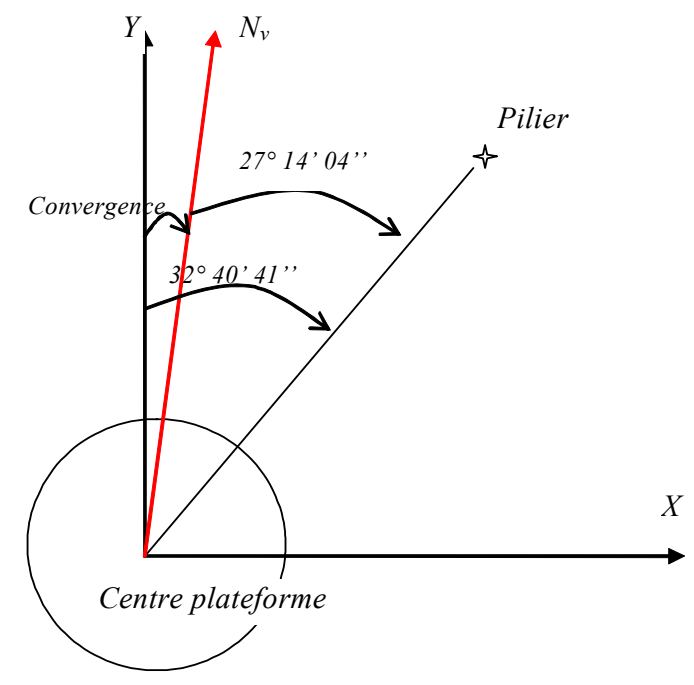

L'axe pilier - centre de la plateforme a été matérialisé et la direction du Nord vrai a été reportée sur la dalle à l'aide d'un rapporteur à alidade réglable à $0,017^{\circ}$ près. Ce rapporteur a servi également à graduer la dalle de proche en proche, tous les $10^{\circ}$. Les directions Nord-Sud et Est-Ouest ont ensuite été vérifiées par repérage à l'aide d'un récepteur GPS (en RTK). L'écart maximum calculé pour l'azimut de l'axe Nord-Sud par rapport au Nord vrai est de $0,44^{\circ}$, alors qu'il est de $0,22^{\circ}$ pour l'axe EstOuest. Pour pointer ces graduations, une flèche rigide a été fixé sur la plateforme et l'axe central du plateau inclinable a été repéré à l'aide d'un théodolite, puis tracé pour avoir un repère pour orienter le courantomètre.

$\mathrm{Au}$ final, si l'on prend en compte l'incertitude sur les directions de référence, celle liée à l'environnement 
magnétique et celle liée à l'inclinaison de la plateforme, on obtient une incertitude élargie de $0,97^{\circ}$ sur les directions de référence.

\section{Essais en cap et correction d'un profileur}

Des essais ont été réalisés avec un profileur AQUAPRO $\mathrm{du}$ constructeur NORTEK. Une première série de mesures a été faite cage centrée, puis décalée vers la gauche puis vers la droite de $50 \mathrm{~cm}$ afin de tester l'homogénéité du champ et la reproductibilité des mesures. Les écarts obtenus se superposent avec une très bonne reproductibilité de $0,19^{\circ}$ (Fig. 3) qui montre d'une part que le placement de la cage sur le plateau importe peu, si son alignement est réalisé avec soin, et d'autre part que le champ magnétique est suffisamment homogène sur le plateau pour ne pas entraîner de biais visibles.

Figure 3: Erreurs en cap du couple Tripode + Aquapro $n^{\circ} \mathrm{AQP} 3356$, axé puis décalé vers la droite et vers la gauche.

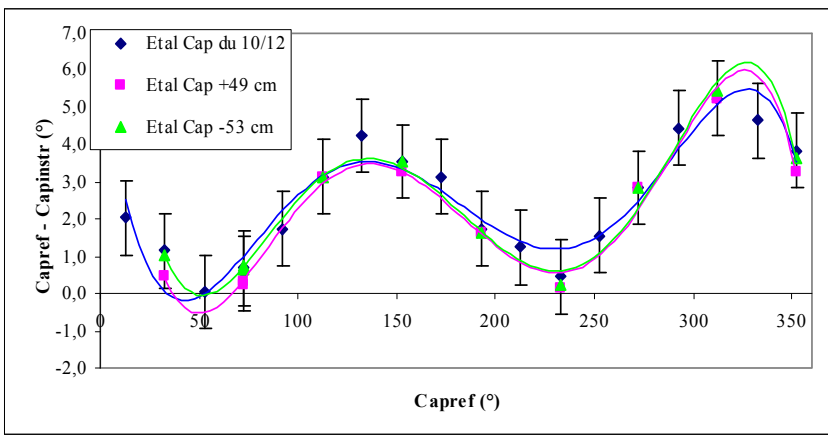

Une publication de J. Fang et al. sortie en 2011 [3] propose une méthode de correction des compas qui repose sur le fait que le modèle d'erreur des compas magnétiques est une ellipsoïde. Cette méthode nécessite cependant d'avoir accès aux valeurs de champ mesurées par les magnétomètres du compas, ce qui n'est pas possible avec les mesureurs de courant.

Il est donc nécessaire de pouvoir corriger directement les mesures de cap. Les erreurs en cap présentent des variations sinusoïdales (Fig. 3). Leur modélisation est possible avec les relations dites 'd'Archibald Smith' [4]. La première prend en compte uniquement l'effet des matériaux magnétiques 'durs' (à champ permanent). La seconde (4) modélise l'effet des matériaux magnétiques 'durs' et 'doux' (qui génèrent un champ en réponse) :

$$
\Gamma=A+B \sin (\Omega)+C \cos (\Omega)+D \cos (2 \Omega)+E \sin (2 \Omega)
$$

L'obtention des coefficients $A, B, C, D$ et $E$ de la relation (4), par une méthode de moindres carrés, permet de modéliser et de corriger parfaitement les écarts mesurés (meilleurs résultats qu'avec un polynôme d'ordre 6). L'écart type d'ajustement obtenu à partir des résidus est de $1^{\prime}$ ordre de $0,35^{\circ}$.

\section{Essais en roulis, tangage et corrections}

Pour ces mesures, la plateforme est inclinée de $2^{\circ}$ en $2^{\circ}$ sur $20^{\circ}$. Puis, le courantomètre est tourné sur son axe de $90^{\circ}$ pour couvrir $\pm 20^{\circ}$ dans les axes de roulis et de tangage.

Concernant les essais réalisés avec l'AQP n 3356, son capteur de tangage présente une courbe d'erreur sinusoïdale. Durant son étalonnage, le capteur de roulis reste constant, par contre, le compas présente un biais qui montre qu'il n'est pas bien compensé en inclinaison par le logiciel de l'instrument.

L'étalonnage en roulis donne les mêmes résultats, l'erreur du compas étant encore plus grande. La correction des erreurs en roulis et tangage peut être faite indifféremment par une relation polynomiale ou sinusoïdale (ajustement à $0,1^{\circ}$ près).

Figure 4 : exemple de résultats obtenus durant un étalonnage en tangage d'un couple Tripode + Aquapro.

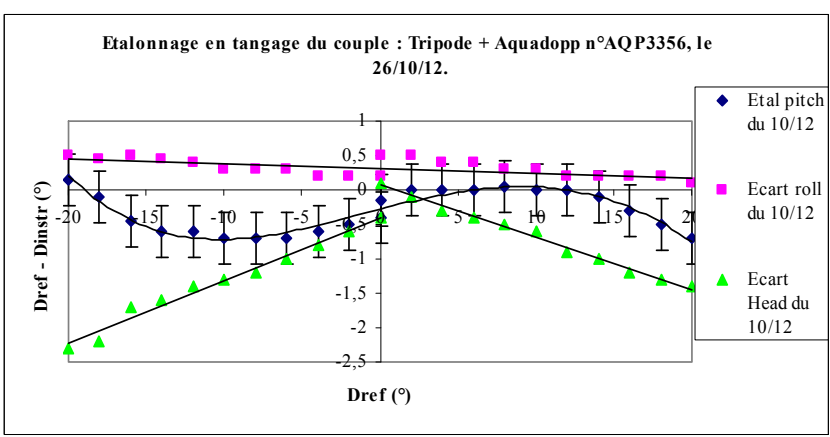

\section{Evaluation des procédures 'd'autocalibration'}

Afin d'essayer de tirer parti des possibilités logicielles des instruments, leurs procédures 'd'auto-étalonnage' ont été testées. Celle du fabricant NORTEK avec l'AQP $\mathrm{n}^{\circ}$ 3356 a été mise en œuvre, sur une aire dépourvue d'anomalies magnétiques, puis on a monté l'instrument sur la plateforme. Le résultat obtenu est plus que décevant. On obtient une courbe dissymétrique avec des écarts aussi importants qu'auparavant (Fig. 5). Par contre, elle peut toujours être corrigée avec la relation (4) (série $\mathrm{G}\left({ }^{\circ}\right)$ de la fig.5).

La procédure du fabricant Teledyne RDI, plus complexe, a été mise en œuvre sur la plateforme. Elle nécessite de réaliser 3 tours avec des inclinaisons différentes. A l'issue, le logiciel RDI indique une erreur totale inférieure à $0,8^{\circ}$, cependant, les mesures sur la plateforme ne donnent pas la même chose : la courbe est de nouveau dissymétrique, décentrée avec des écarts maximum de $-3^{\circ}$. 
Figure 5 : réponse obtenue après application d'une procédure 'd'autocalibration'.

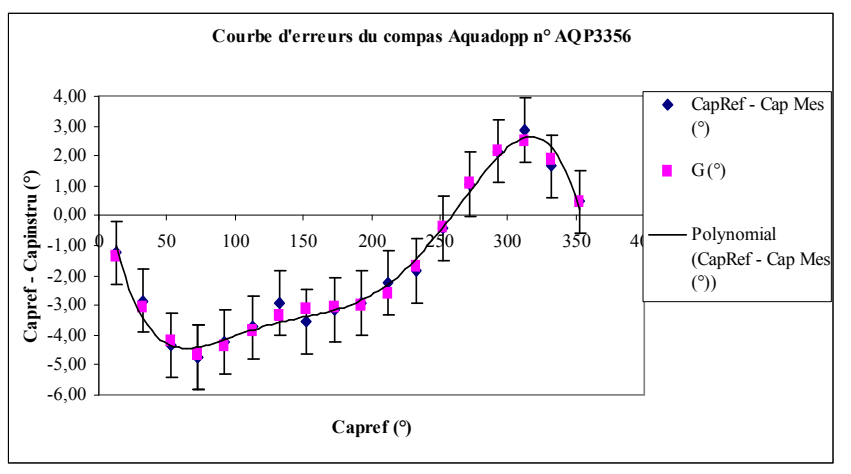

\section{Conclusion et perspectives}

Les courantomètres et profileurs de courant sont des instruments complexes dont la maîtrise métrologique reste un défi (compte tenu du nombre de variables à contrôler).

Si des étalonnages ou des intercomparaisons en vitesse peuvent être réalisés dans certains cas, l'étude de leurs erreurs angulaires est un domaine qui a été jusqu'à présent peu abordé du fait :

- du manque d'informations (plus ou moins volontaire) venant des constructeurs d'instruments ;

- de la confiance des utilisateurs dans leurs instruments ;

- du manque de moyens de tests et d'étalonnage des capteurs d'angle et d'orientation.

Cette plateforme d'étalonnage des compas et capteurs d'inclinaison va permettre de mieux connaître et de corriger les biais de ces capteurs.

Elle pourrait avoir d'autres applications ou intérêts potentiels. Connaître la direction des courants avec exactitude doit permettre de modéliser et d'améliorer le rendement des hydroliennes. Celui-ci est généralement fonction de l'angle d'incidence du courant sur la turbine.

Figure 6 : schéma d'hydrolienne de la société SABELLA s.a.s..

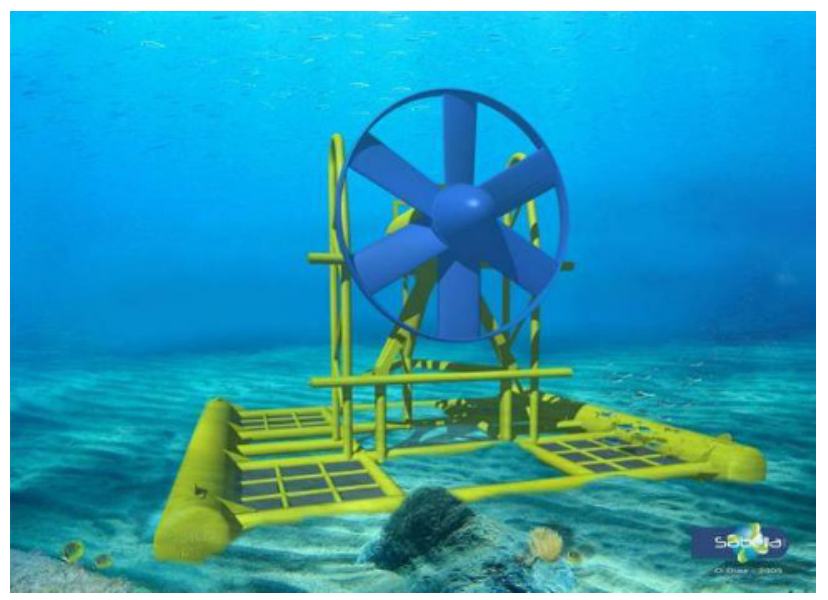

\section{References}

1. M. J. Caruso, 'Application of magnetoresistive sensors in navigation systems', Sensors Actuators, 1220, 15-21, (1997).

2. M. Le Menn, M. Le Goff, 'A method for absolute calibration of compasses', Measurement Science and Technology, 18, 1614-1621, (2007).

3. J. Fang, H. Sun, J. Cao, X. Zhang, Y. Tao, 'A novel calibration method of magnetic compass based on ellipsoid fitting', IEEE Transaction on Instrumentation and Measurement, 60, 6, 2053 - 2061, (2011).

4. W. Denne, Magnetic compass deviation and correction, $3^{\text {rd }}$ edition, Brown, Son and Fergusson, 165 pp, (1998). 